\title{
Degenerate Backward wave Optical Parametric Oscillator
}

\author{
Patrick Mutter ${ }^{1}$, Andrius Zukauskas ${ }^{1}$, Anne-Lise Viotti ${ }^{2,3}$, Valdas Pasiskevicius ${ }^{1}$ and Carlota \\ Canalias ${ }^{1}$ \\ 1. Department of Applied Phzsics, Royal Institute of Technology, Roslagstullbacken 21, 10691, Stockholm, Sweden \\ 2. Deutsches Elektronen-Synchrotron DESY, Notkestraße 85, 22607 Hamburg, Germany \\ 3. Department of Physics, Lund University, P.O. Box 118, SE-221 00 Lund, Sweden
}

Backward wave optical parametric oscillators (BWOPO) are a special class of nonlinear optical devices with unique properties, due to the fact, that the pump photon is split in two photons propagating in opposite directions. The counter-propagating nature of the interaction automatically establishes positive feedback, without any external cavity, making the system very robust and insensitive to misalignment. Furthermore, the forward wave inherits the phase modulation of the pump, while the backward generated wave is inherently narrow [1]. Although the concept of BWOPO was proposed in the 1960s [2], the fist experimental demonstration had to wait until 2007 [3]. This long delay is ascribed to fact that quasi-phase matched (QPM) structures with sub-um periodicities are required to compensate for the large phase-mismatch between the counter-propagating waves. Thanks to the recent progress in periodic poling based on coercive field engineering, several BWOPO interactions with ps and ns pump pulses have been achieved, demonstrating efficient single pass configuration with conversion efficiencies up to $50 \%$ at a mJ output level [4]. BWOPO operating at degeneracy, especially in the telecommunications band, is of special interest due to the possibility to have phase locking inherent to degenerate parametric processes which can be used for generation of biphoton states, a resource for quantum communications and quantum sensing [5,6]. Efficient BWOPO operation in the spectral range of $1.5 \mu \mathrm{m}-1.6 \mu \mathrm{m}$, requires first-order QPM, which can be realized in periodic nonlinear structures with a periodicity of $\sim 400 \mathrm{~nm}$, i.e., close to half of the pump wavelength in vacuum.

In this work, we take advantage of our coercive-field engineering technique [7] to fabricate $435 \mathrm{~nm}$-period in 1 $\mathrm{mm}$ thick Rb-doped $\mathrm{KTiOPO}_{4}$ (PPRKTP) crystals. The resulting domain pattern shows excellent uniformity over the entire crystal aperture and a duty cycle close to $50 \%$ (see Fig. 1a)) To the best of our knowledge, this poling period marks the shortest QPM period ever fabricated in ferroelectric crystals of comparable aperture. The crystals are used to demonstrate the first realization of a degenerate BWOPO, generating counter-propagating signal and idler in the spectral band of optical communications.
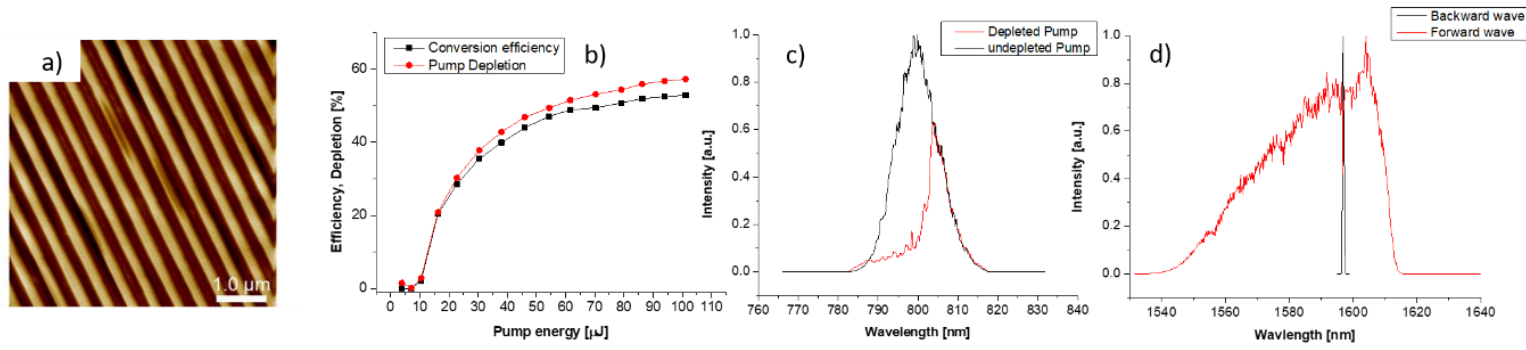

Fig. 1 a) Piezo-force microscope image of one of the polar faces of PPRKTP crystal, b) Combined forward-and backward signal conversion efficiency (black) and pump depletion (red symbols), c) Undepleted Pump (black) and depleted pump (red) d) Forward (red)- and backward wave (black) spectra

The PPRKTP crystal was pumped by a Ti:Sapphire regenerative amplifier delivering 240 ps-long chirped pulses at a central wavelength of $799 \mathrm{~nm}$ and a spectral bandwidth of about $10 \mathrm{~nm}$ at $1 \mathrm{kHz}$ repetition rate. The laser beam was focused into the crystal to a beam radius of $51 \mathrm{um}$. The BWOPO process started at a threshold of 265 $\mathrm{MW} / \mathrm{cm}^{2}$. The conversion efficiency reached $53 \%$ at a pump energy of $101 \mu \mathrm{J}$ (see Fig. $1 \mathrm{~b}$ )). Fig c) and d) show the undepleted and depleted pump, the forward wave and backward wave spectra, respectively. As expected, the backward wave was narrowband $(0.65 \mathrm{~nm})$ centered at $1597 \mathrm{~nm}$ and the forward wave is a spectral replica of the pump. Note that at the degeneracy point $(1597 \mathrm{~nm})$ the forward wave show a dip in their spectrum. This is attributed to phase-matched second harmonic generation by the counter-propagating BWOPO signal and idler. This explains the small discrepancy between the pump depletion and the measured BWOPO efficiency. These cascaded interactions bring such functionalities as nonclassical squeezed light generation as well as give opportunity to explore counter-propagating comb generation [8].

\section{References}

[1] G. Strömqvist, V. Pasiskevicius, C. Canalias, and C. Montes, "Coherent phase-modulation transfer in counterpropagating parametric down-conversion," Phys. Rev. A - At. Mol. Opt. Phys., vol. 84, no. 2, pp. 1-4, 2011.

[2] S. E. Harris, "Proposed backward wave oscillation in the infrared," Appl. Phys. Lett., vol. 9, no. 3, pp. 114-116, 1966.

[3] C. Canalias and V. Pasiskevicius, "Mirrorless optical parametric oscillator," pp. 459-462, 2007.

[4] R. S. Coetzee, A. Zukauskas, C. Canalias, and V. Pasiskevicius, "Low-threshold, mid-infrared backward-wave parametric oscillator with periodically poled Rb:KTP," APL Photonics, vol. 3, no. 7, 2018.

[5] K.-H. Luo et al., "Counter-propagating photon pair generation in a nonlinear waveguide," Opt. Express, vol. 28, no. 3, p. 3215, 2020.

[6] C. S. Chuu and S. E. Harris, "Ultra-bright backward wave biphoton source,", "Phys. Rev. A 83, 061803(R) (2011)

[7] Liljestrand, F. Laurell, and C. Canalias, "Periodic poling of Rb-doped KTiOPO4" Opt. Express 24,14682, (2016)

[8] I. Ricciardi, et al "Frequency comb generation in quadratic nonlinear media," Phys. Rev. A, 91, 063839 (2015). 